\title{
FACTORIALITY OF BOŻEJKO-SPEICHER VON NEUMANN ALGEBRAS
}

\author{
PIOTR ŚNIADY
}

\begin{abstract}
We study the von Neumann algebra generated by q-deformed Gaussian elements $l_{i}+l_{i}^{*}$ where operators $l_{i}$ fulfill the q-deformed canonical commutation relations $l_{i} l_{j}^{*}-q l_{j}^{*} l_{i}=\delta_{i j}$ for $-1<q<1$. We show that if the number of generators is finite, greater than some constant depending on $\mathrm{q}$, it is a $\mathrm{II}_{1}$ factor which does not have the property $\Gamma$. Our technique can be used for proving factoriality of many examples of von Neumann algebras arising from some generalized Brownian motions, both for type $\mathrm{II}_{1}$ and type III case.
\end{abstract}

\section{INTRODUCTION}

In 1970 Frisch and Bourret [FB70] considered commutation relation

$$
l_{\phi} l_{\psi}^{*}-q l_{\psi}^{*} l_{\phi}=(\phi, \psi)
$$

fulfilled by annihilation operator $l$ and its adjoint creation operator $l^{*}$ for $q \in \mathbb{R}$. Annihilation and creation operators are indexed by elements of a fixed real Hilbert space $\mathcal{H}_{\mathbb{R}}$ and they act on a complex Hilbert space $\mathcal{F}$, called Fock space. There is a distinguished unital vector $\Omega \in \mathcal{F}$, called vacuum, such that $l_{\phi} \Omega=0$ for every $\phi \in \mathcal{H}_{\mathbb{R}}$. It was a long time until Bożejko and Speicher [BS94] showed in 1994 the existence of the operators considered by Frisch and Bourret for all $-1<\mathrm{q}<1$.

Frisch and Bourret were studying generalized Gaussian variables $\mathrm{L}_{\phi}=$ $l_{\phi}+l_{\phi}^{*}$; it turns out that the algebra $\Gamma_{\mathrm{q}}$ generated by $\left(\mathrm{L}_{\phi}\right)$ can be equipped with a tracial state $a \mapsto\langle\Omega, a \Omega\rangle$. The motivation for studying such Gaussian variables is that if $q=1$ then (11) coincides with the canonical commutation relations and hence $\left(L_{\phi}\right)$ can be identified with a family of classical Gaussian random variables; for $q=-1$ relation (1) coincides with the canonical anticommutation relations; furthermore it turned out much later that for $\mathrm{q}=0$ relation (11) coincides with the free relation and hence $\left(\mathrm{L}_{\phi}\right)$ is a family of Voiculescu semicircular elements [VDN92].

In this article we will study the von Neumann algebra $\Gamma_{\mathrm{q}}$ generated by $q$-deformed Gaussian variables $L_{\phi}$. Since for $q=0$ these von Neumann

Research supported by State Committee for Scientific Research (KBN) grant 2 P03A 00723. 
algebras are isomorphic to the free group factors, we can consider the general case as a 'smooth' deformation of this eminent case. Not too much is known about $\Gamma_{\mathrm{q}}$, in particular it is not clear if it is always isomorphic to the free group factors. Bożejko and Speicher [BS94] showed that under certain conditions $\Gamma_{\mathrm{q}}$ is non-injective; recently Nou [Nou02] showed that it is enough to assume that $-1<q<1$ and $\operatorname{dim} \mathcal{H}_{\mathbb{R}} \geq 2$. Recently Shlyakhtenko [Shl03] showed that if $|\mathrm{q}|<\sqrt{2}-1$ and $\operatorname{dim} \mathcal{H}_{\mathbb{R}} \geq 2$ then algebras $\Gamma_{\mathrm{q}}$ are solid (cf. [Oza03]) and if they are factors then they do not have the property $\Gamma$.

Bożejko, Kümmerer and Speicher [BKS97] showed that if the number of generators is infinite (i.e. if $\operatorname{dim} \mathcal{H}_{\mathbb{R}}=\infty$ ) then for every $-1<q<1$ the algebra $\Gamma_{\mathrm{q}}$ is a $\mathrm{II}_{1}$ factor. In this article we show that this result remains true if the number of generators is finite, greater than some constant depending on $\mathrm{q}$ and that this factor does not have the property $\Gamma$. We also point out that the same proof can be used for proving the analogous result for many other von Neumann algebras, both finite and infinite.

\section{NOTATIONS}

If not stated otherwise, all results presented in this section are due to Bożejko and Speicher [BS94].

2.1. Fock space. Let $\mathcal{H}_{\mathbb{R}}$ be a real Hilbert space equipped with a bilinear scalar product $(\cdot, \cdot)$; we will denote its complexification by $\mathcal{H}$ and the corresponding sesquilinear scalar product by $\langle\cdot, \cdot\rangle$. Let furthermore $-1<\mathrm{q}<1$ be fixed. For integer $n \geq 0$ we introduce an operator $P^{(n)}: \mathcal{H}^{\otimes n} \rightarrow \mathcal{H}^{\otimes n}$ given by

$$
P^{(n)}\left(\psi_{1} \otimes \cdots \otimes \psi_{n}\right)=\sum_{\sigma \in S_{n}} q^{\text {inv } \sigma} \psi_{\sigma(1)} \otimes \cdots \otimes \psi_{\sigma(n)},
$$

where inv $\sigma$ is the number of inversions in $\sigma$, i.e. the number of pairs $(i, j)$ such that $1 \leq i<j \leq n$ and $\sigma(i)>\sigma(j)$. Operator $P^{(n)}$ is strictly positive, therefore we can equip $\mathcal{H}^{\otimes n}$ with a new scalar product

$$
\langle\Phi, \Psi\rangle_{\mathrm{q}}=\left\langle\Phi, \mathrm{P}^{(\mathrm{n})} \Psi\right\rangle
$$

for $\Phi, \Psi \in \mathcal{H}^{\otimes \mathrm{n}}$ where $\langle\cdot, \cdot\rangle$ denotes the standard scalar product on $\mathcal{H}^{\otimes \mathrm{n}}$. In the following by $\mathcal{H}^{\otimes n}$ we will mean the $n$-fold tensor product equipped with the standard scalar product $\langle\cdot, \cdot\rangle$ and by $\mathcal{H}_{\mathbf{q}}^{\otimes n}$ the $n$-fold tensor product equipped with the scalar product $\langle\cdot, \cdot\rangle_{\mathrm{q}}$.

The q-Fock space $\mathcal{F}$ is a complex Hilbert space defined by

$$
\mathcal{F}=\mathcal{F}\left(\mathcal{H}_{\mathbb{R}}\right)=\bigoplus_{n \geq 0} \mathcal{H}_{q}^{\otimes n}
$$


where the term $\mathcal{H}_{\mathrm{q}}^{\otimes 0}$ should be understood as one-dimensional space $\mathbb{C} \Omega$ for some unital vector $\Omega$. By $\mathcal{H} \otimes \mathcal{F}$ we will mean the tensor product of Hilbert spaces $\mathcal{H}$ and $\mathcal{F}$ equipped with the canonical scalar product of the scalar product in $\mathcal{H}$ and the scalar product in $\mathcal{F}$.

We consider the Hilbert space

$$
\mathcal{F}^{+}=\bigoplus_{n \geq 1} \mathcal{H}_{\mathrm{q}}^{\otimes n}=\Omega^{\perp} \subset \mathcal{F}
$$

and the map

$$
j: \mathcal{H} \otimes \mathcal{F} \rightarrow \mathcal{F}^{+}
$$

given by the trivial mapping of Hilbert spaces

$$
\phi \otimes\left(\psi_{1} \otimes \cdots \otimes \psi_{n}\right) \mapsto \phi \otimes \psi_{1} \otimes \cdots \otimes \psi_{n} .
$$

Please note that the map (3) is not as trivial as it might appear since the scalar products are different on the domain and on the range.

Proposition 2.1. For every $-1<\mathrm{q}<1$ there exist constants $\mathrm{C}_{1}, \mathrm{C}_{2}$ such that for every choice of the real Hilbert space $\mathcal{H}_{\mathbb{R}}$

$$
\|\mathrm{j}\| \leq \mathrm{C}_{1}, \quad\left\|\mathrm{j}^{-1}\right\| \leq \mathrm{C}_{2} .
$$

Similar inequalities hold for the right trivial mapping $\mathcal{F} \otimes \mathcal{H} \rightarrow \mathcal{F}^{+}$given by $\left(\psi_{1} \otimes \cdots \otimes \psi_{n}\right) \otimes \phi \mapsto \psi_{1} \otimes \cdots \otimes \psi_{n} \otimes \phi$.

Proof. Bożejko and Speicher showed that

$$
\mathrm{P}^{(\mathrm{n}+1)} \leq \frac{1}{1-|\mathrm{q}|}\left(1 \otimes \mathrm{P}^{(\mathrm{n})}\right)
$$

and Bożejko [Boż98] showed that there exists a positive constant $\omega(q)$ such that for each $n$ we have

$$
1 \otimes P^{(n)} \leq \frac{1}{\omega(q)} P^{(n+1)}
$$

2.2. Annihilation and creation operators. For $\phi \in \mathcal{H}_{\mathbb{R}}$ we consider left and right creation operators $l_{\phi}^{*}, r_{\phi}^{*}: \mathcal{F} \rightarrow \mathcal{F}$ defined on elementary tensors by

$$
\begin{aligned}
& l_{\phi}^{*}\left(\psi_{1} \otimes \cdots \otimes \psi_{n}\right)=\phi \otimes \psi_{1} \otimes \cdots \otimes \psi_{n}, \\
& r_{\phi}^{*}\left(\psi_{1} \otimes \cdots \otimes \psi_{n}\right)=\psi_{1} \otimes \cdots \otimes \psi_{n} \otimes \phi .
\end{aligned}
$$

We also consider their adjoints: left and right annihilation operators

(6) $l_{\phi}\left(\psi_{1} \otimes \cdots \otimes \psi_{n}\right)=\sum_{1 \leq i \leq n} q^{i-1}\left(\phi, \psi_{i}\right) \psi_{1} \otimes \cdots \otimes \widehat{\psi}_{i} \otimes \cdots \otimes \psi_{n}$, 
(7) $r_{\phi}\left(\psi_{1} \otimes \cdots \otimes \psi_{n}\right)=\sum_{1 \leq i \leq n} q^{n-i}\left(\phi, \psi_{i}\right) \psi_{1} \otimes \cdots \otimes \widehat{\psi_{i}} \otimes \cdots \otimes \psi_{n}$ where $\widehat{\psi_{i}}$ denotes an omitted factor and $n \geq 1$. The case $n=0$ should be understood as $l_{\phi} \Omega=r_{\phi} \Omega=0$. All creation and annihilation operators are bounded.

Remark. Please note that in the definition of the annihilation operators the natural extension of the bilinear scalar product $(\cdot, \cdot)$ was used and not the sesquilinear scalar product $\langle\cdot, \cdot\rangle$.

2.3. Von Neumann algebra. Let $\Gamma_{\mathrm{q}}$ denote the von Neumann algebra generated by the family of selfadjoint operators $L_{\phi}: \mathcal{F} \rightarrow \mathcal{F}$ given by

$$
\mathrm{L}_{\phi}=\mathrm{l}_{\phi}+\mathrm{l}_{\phi}^{*}
$$

for $\phi \in \mathcal{H}_{\mathbb{R}}$. Algebra $\Gamma_{\mathrm{q}}$ is equipped with a faithful tracial state $\chi \mapsto$ $\langle\Omega, x \Omega\rangle$. Vector $\Omega$ is separating and cyclic for $\Gamma_{\mathrm{q}}$; let $\mathrm{J}: \mathcal{F} \rightarrow \mathcal{F}$ denote the canonical involution. Then $\mathrm{JL}_{\phi} \mathrm{J}=\mathrm{R}_{\phi}$, where

$$
R_{\phi}=r_{\phi}+r_{\phi}^{*}
$$

for $\phi \in \mathcal{H}_{\mathbb{R}}$. Operators $\left(\mathrm{L}_{\phi}\right)$ commute with operators $\left(\mathrm{R}_{\psi}\right)[\mathrm{BX} 00]$.

\section{THE MAIN RESULT}

Let us fix some finite-dimensional real Hilbert subspace $\mathcal{H}_{\mathbb{R}}^{\prime} \subseteq \mathcal{H}_{\mathbb{R}}$ and let $e_{1}, \ldots, e_{\mathrm{d}}$ be an orthonormal basis of $\mathcal{H}_{\mathbb{R}}^{\prime}$. We denote by $\mathcal{H}^{\prime}$ the complexification of $\mathcal{H}_{\mathbb{R}}^{\prime}$.

In Section 4 we will define a certain operator $M$. It is enough to know now that $|M|^{2}: \mathcal{F} \rightarrow \mathcal{F}$ is given by

$$
|M|^{2}=M^{*} M=\sum_{i}\left(L_{e_{i}}-R_{e_{i}}\right)^{2} .
$$

In Section 4 we will show the following.

Proposition 3.1. $\mathbb{C} \Omega$ belongs to the kernel of $|\mathrm{M}|$.

For every $-1<\mathrm{q}<1$ there exists $\mathrm{d}_{0}$ such that if $\mathrm{d} \geq \mathrm{d}_{0}$ then the restriction of $|\mathrm{M}|$ to the space $\mathcal{F}^{+}$is strictly positive in the sense that

$$
|M| \geq \epsilon>0
$$

holds for some $\epsilon$.

The above result has an immediate consequence.

Theorem 3.2. Let $\mathrm{d}_{0}$ be the constant from Proposition 3.1 If $\operatorname{dim} \mathcal{H}_{\mathbb{R}} \geq \mathrm{d}_{0}$ (the case $\operatorname{dim} \mathcal{H}_{\mathbb{R}}=\infty$ is allowed) then $\Gamma_{\mathrm{q}}$ is a II factor which does not have the property $\Gamma$. 
Proof. We choose finite-dimensional $\mathcal{H}_{\mathbb{R}}^{\prime} \subseteq \mathcal{H}_{\mathbb{R}}$ in such a way that $\operatorname{dim} \mathcal{H}_{\mathbb{R}}^{\prime} \geq$ $\mathrm{d}_{0}$.

Let $a \in \Gamma_{\mathrm{q}}$ be central; it follows that a commutes with $|\mathrm{M}|$ hence $|\mathrm{M}| \mathrm{a} \Omega=$ $a|M| \Omega=0$ and $a \Omega$ belongs to the kernel of $|M|$. Proposition 3.1 implies that $a \Omega \in \mathbb{C} \Omega$. Since $\Omega$ is separating it follows that $a \in \mathbb{C}$ hence $\Gamma_{\mathrm{q}}$ is a factor.

The following observation was pointed out to me by Dimitri Shlyakhtenko. The operator $|M|$ belongs to the $C^{*}$-algebra generated by $\Gamma_{\mathrm{q}}$ and $\mathrm{J} \Gamma_{\mathrm{q}} J$. Proposition 3.1 shows that the kernel of $|M|$ is equal to $\mathbb{C} \Omega$ and that the zero eigenvalue is separated. It follows that the orthogonal projection $\mathcal{F} \rightarrow \mathbb{C} \Omega$ belongs to $\mathrm{C}^{*}\left(\Gamma_{\mathrm{q}}, J \Gamma_{\mathrm{q}} J\right)$. By the result of Connes [Con76] it follows that $\Gamma_{\mathrm{q}}$ does not have the property $\Gamma$.

\section{INEQUALITIES}

We define $M: \mathcal{F} \rightarrow \mathcal{H} \otimes \mathcal{F}$ by

$$
M(\Phi)=\sum_{i} e_{i} \otimes\left(L_{e_{i}}(\Phi)-R_{e_{i}}(\Phi)\right) .
$$

It is easy to check that $(8)$ is indeed fulfilled. We also define auxiliary maps $\mathrm{m}, \mathrm{m}^{\dagger}: \mathcal{F} \rightarrow \mathcal{H} \otimes \mathcal{F}$ given by

$$
\begin{aligned}
& m(\Phi)=\sum_{i} e_{i} \otimes\left(l_{e_{i}}(\Phi)-r_{e_{i}}(\Phi)\right), \\
& m^{\dagger}(\Phi)=\sum_{i} e_{i} \otimes\left(l_{e_{i}}^{*}(\Phi)-r_{e_{i}}^{*}(\Phi)\right) .
\end{aligned}
$$

Clearly $M=m+m^{\dagger}$. Please note that the definitions of the above operators do not depend on the choice of the orthonormal basis $\left(e_{i}\right)$.

Lemma 4.1. The norm of $\mathrm{m}$ fulfills

$$
\|\mathrm{m}\| \leq 2 \mathrm{C}_{1}
$$

where $\mathrm{C}_{1}$ is the constant from Proposition 2.1

Proof. Consider the map $\mathrm{m}_{l}: \mathcal{F}^{+} \rightarrow \mathcal{H} \otimes \mathcal{F}$ given by

$$
m_{l} \Phi=\sum_{i} e_{i} \otimes\left(l_{e_{i}} \Phi\right)
$$

Its adjoint $\mathrm{m}_{\mathrm{l}}^{*}: \mathcal{H} \otimes \mathcal{F} \rightarrow \mathcal{F}^{+}$is given by

$$
\mathrm{m}_{\mathrm{l}}^{*}(\phi \otimes \Phi)=\sum_{i}\left(e_{i}, \phi\right) \mathrm{l}_{e_{i}}^{*} \Phi=\mathrm{j}\left[\left(\Pi_{\mathcal{H}^{\prime}} \phi\right) \otimes \Phi\right]
$$

where $j$ denotes the trivial map (3) and $\Pi_{\mathcal{H}^{\prime}}: \mathcal{H} \rightarrow \mathcal{H}^{\prime}$ is the orthogonal projection. Therefore $\left\|m_{l}\right\|=\left\|m_{l}^{*}\right\| \leq\|j\| \leq C_{1}$. 
Similar inequality can be obtained for the right annihilator

$$
\mathrm{m}_{\mathrm{r}} \Phi=\sum_{i} e_{\mathrm{i}} \otimes\left(\mathrm{r}_{\mathrm{e}_{\mathrm{i}}} \Phi\right)
$$

it remains to notice that $m=m_{l}+m_{r}$.

Lemma 4.2. The restriction of $\left|\mathrm{m}^{\dagger}\right|$ to the space $\mathcal{F}^{+}$fulfils

$$
\left|\mathrm{m}^{\dagger}\right| \geq \frac{\mathrm{d}-\mathrm{C}_{1} \mathrm{C}_{2}}{\mathrm{C}_{2} \sqrt{\mathrm{d}}}
$$

Proof. Consider the map S: $\mathcal{F}^{+} \rightarrow \mathcal{F}^{+}$:

$$
S\left(\phi_{1} \otimes \cdots \otimes \phi_{n}\right)=\phi_{2} \otimes \cdots \otimes \phi_{n} \otimes\left(\Pi_{\mathcal{H}^{\prime}} \phi_{1}\right),
$$

where $\Pi_{\mathcal{H}^{\prime}}: \mathcal{H} \rightarrow \mathcal{H}^{\prime}$ denotes the orthogonal projection. Proposition 2.1 implies that $\|S\| \leq \mathrm{C}_{1} \mathrm{C}_{2}$.

Consider the map $\tilde{f}: \mathcal{H} \otimes \mathcal{H} \otimes \mathcal{F} \rightarrow \mathcal{F}$ given by

$$
\begin{aligned}
\tilde{f}\left(\phi \otimes \psi_{1} \otimes\left(\psi_{2} \otimes \cdots \otimes \psi_{n}\right)\right) & = \\
& \left\langle\sum_{k} e_{k} \otimes e_{k}, \phi \otimes \psi_{1}\right\rangle \psi_{2} \otimes \cdots \otimes \psi_{n} ;
\end{aligned}
$$

its norm is equal to $\left\|\sum_{k} e_{k} \otimes e_{k}\right\|=\sqrt{d}$. From Proposition 2.1 it follows that $f: \mathcal{H} \otimes \mathcal{F}^{+} \rightarrow \mathcal{F}$ given by

$$
f\left(\phi \otimes\left(\psi_{1} \otimes \cdots \otimes \psi_{n}\right)\right)=\left\langle\sum_{k} e_{k} \otimes e_{k}, \phi \otimes \psi_{1}\right\rangle \psi_{2} \otimes \cdots \otimes \psi_{n}
$$

fulfills $\|f\| \leq C_{2} \sqrt{d}$ (because the only difference between the maps $f$ and $\tilde{f}$ is the choice of the norm on the domain).

It is easy to check that for $\Phi \in \mathcal{F}^{+}$

$$
f \mathrm{~m}^{\dagger} \Phi=(\mathrm{d}-\mathrm{S}) \Phi
$$

It follows

$$
C_{2} \sqrt{d}\left\|m^{\dagger}(\Phi)\right\| \geq\left\|f m^{\dagger}(\Phi)\right\|=\|(d-S) \Phi\| \geq\left(d-C_{1} C_{2}\right)\|\Phi\| .
$$

Proof of Proposition 3.1 It is easy to check that $\Omega$ belongs to the kernel of $|\mathrm{M}|^{2}$.

Observe that if the restrictions of the operators to the space $\mathcal{F}^{+}$fulfill

$$
\left|\mathrm{m}^{\dagger}\right|>\|\mathrm{m}\|
$$

then the restriction of $|M|=\left|\mathrm{m}^{\dagger}+\mathrm{m}\right|$ to $\mathcal{F}^{+}$is strictly positive. Estimates from the above Lemmas finish the proof. 
Remark. For the case $\mathrm{q}=0$ one can take $\mathrm{C}_{1}=\mathrm{C}_{2}=1$ and Theorem 3.2 shows factoriality of $\Gamma_{0}$ for $\operatorname{dim} \mathcal{H}_{\mathbb{R}} \geq 6$ while we know (from the free group construction) that it is valid also for $\operatorname{dim} \mathcal{H}_{\mathbb{R}} \geq 2$ therefore our result is far from being fully satisfactory.

\section{GeneralizATIONS}

A careful reader might easily observe that in Sections 3 and 4 we used only very few properties of Bożejko-Speicher algebras. In particular, we did not use the exact form of the symmetrizer (2) and of the annihilation operators (6), (7), nor the existence of the tracial state $a \mapsto\langle\Omega, a \Omega\rangle$.

It follows that our proof can be used in a more general context to proof factoriality (both in type $\mathrm{II}_{1}$ and type III case) of some von Neumann algebras arising from some generalized Brownian motions for which an analogue of Proposition 2.1 holds.

It is easy to find a whole zoo of examples since already Bożejko and Speicher in their original article [BS94] considered symmerizations arising from Yang-Baxter operators which are much more general than (2); Królak [Krö00] generalized further these results. Also Hiai [Hia03] generalized results of Shlyakhtenko [Sh197] and constructed von Neumann algebras which are q-analogues of free Araki-Woods algebras. It was pointed out to me by Mădălin Guţă that the generalized Brownian motions considered in the work [GM02] might also provide appropriate examples.

\section{ACKNOWLEDGMENTS}

I thank Marek Bożejko for introducing me into the subject and for many discussions. I thank Benoît Collins for teaching me the right way of thinking about tensors. I also thank Mădălin Guţă, Fumio Hiai, Dimitri Shlyakhtenko and the Reviewer for many helpful remarks.

Research supported by State Committee for Scientific Research (KBN) grant No. 2 P03A 007 23. The research was conducted in Syddansk Universitet (Odense, Denmark) and Banach Center (Warszawa, Poland) on a grant funded by European Post-Doctoral Institute for Mathematical Sciences.

\section{REFERENCES}

[BKS97] Marek Bożejko, Burkhard Kümmerer, and Roland Speicher. q-Gaussian processes: non-commutative and classical aspects. Comm. Math. Phys., 185(1):129-154, 1997.

[Boż98] Marek Bożejko. Completely positive maps on Coxeter groups and the ultracontractivity of the q-Ornstein-Uhlenbeck semigroup. In Quantum probability (Gdańsk, 1997), pages 87-93. Polish Acad. Sci., Warsaw, 1998. 
[BS94] Marek Bożejko and Roland Speicher. Completely positive maps on Coxeter groups, deformed commutation relations, and operator spaces. Math. Ann., 300(1):97-120, 1994.

[BX00] Marek Bożejko and Quanhua Xu. Factoriality and second quantization. Unpublished, 2000.

[Con76] A. Connes. Classification of injective factors. Cases $\mathrm{II}_{1}, \mathrm{II}_{\infty}, \mathrm{III}_{\lambda}, \lambda \neq 1$. Ann. of Math. (2), 104(1):73-115, 1976.

[FB70] U. Frisch and R. Bourret. Parastochastics. J. Mathematical Phys., 11:364-390, 1970.

[GM02] Mădălin Guţă and Hans Maassen. Generalised Brownian motion and second quantisation. J. Funct. Anal., 191(2):241-275, 2002.

[Hia03] Fumio Hiai. q-deformed Araki-Woods factors. In J.-M. Combes et al. (eds.) Operator Algebras and Mathematical Physics, pages 169-202. Theta, Bucharest, 2003.

[Krö00] Ilona Krỏlak. Wick product for commutation relations connected with YangBaxter operators and new constructions of factors. Comm. Math. Phys., 210(3):685-701, 2000.

[Nou02] Alexander Nou. Non injectivity of the q-deformed von neumann algebras. Preprint, 2002.

[Oza03] Narutaka Ozawa. Solid von Neumann Algebras. Preprint math.OA/0302082, 2003.

[Sh197] Dimitri Shlyakhtenko. Free quasi-free states. Pacific J. Math., 177(2):329-368, 1997.

[Shl03] Dimitri Shlyakhtenko. Some estimates for non-microstates free entropy dimension, with applications to q-semicircular families. Preprint math. OA/0308093 2003.

[VDN92] D. V. Voiculescu, K. J. Dykema, and A. Nica. Free random variables. American Mathematical Society, Providence, RI, 1992. A noncommutative probability approach to free products with applications to random matrices, operator algebras and harmonic analysis on free groups.

InSTITUTE OF MATHEMATICS, UNIVERSity OF WROCLAW, PL. GRUNWALDZKi 2/4, 50-384 WROCLAW, POLAND

E-mail address: Piotr.Sniady@math.uni.wroc.pl 